\title{
PROPERTIES OF GLOBULAR CLUSTER SYSTEM: PRIMORDIAL OR EVOLUTIONAL?
}

\author{
VLADIMIR SURDIN \\ Sternberg Astronomical Institute \\ 13, Universitetskij Prospect \\ Moscow, 119899, Russia \\ e-mail: surdin@sai.msu.su
}

\begin{abstract}
Some observable relationships between globular cluster parameters appear as a result of long time dynamical evolution of the cluster system. These relationships are inapplicable to the studies of the globular clusters origin.
\end{abstract}

\section{Introduction}

There are some well known relations between dynamical parameters of Galactic globular clusters and their galactocentric distances $\left(R_{g}\right)$. If this relations are primordial, we can obtain an important knowledge on physical conditions at the globular cluster formation epoch. But if the relations are evolutional, we do not have such possibility. Any way, we must understand reasons for each particular relation to solve the problem of globular cluster formation. It is particularly important to find some invariant relations which keep stable along the evolutional path of the cluster system.

The values of $\left(R_{g}\right)$ and the diameters containing half of the cluster mass/luminosity in projection $\left(D_{0.5}\right)$ are mostly invariable characteristics of them. Therefore these are used for the comparison of the predictions of the cluster formation theory with observational data.

However it is easy to show that, in spite of a relative evolutional stability of the particular values of $R_{g}$ and $D_{0.5}$, the relation between them (Fig. 1)

$$
D_{0.5} \propto R_{g}^{1 / 2}
$$




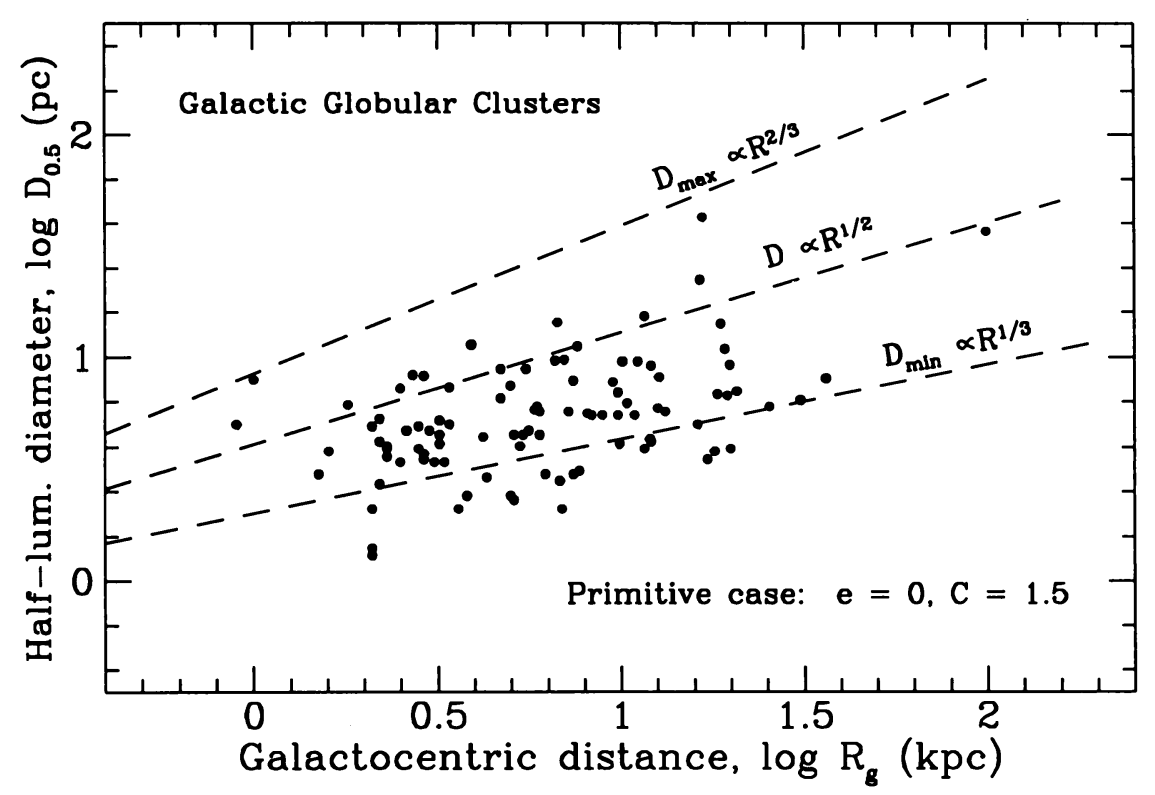

Figure 1. Distribution of globular clusters on $R_{g}$ v. $D_{0.5}$ plot; data by van den Bergh at al., 1991. Theoretical lines of limiting diameters and predicted correlation are shown for the simplest case of circular orbits and equal concentration parameters.

for the globular cluster system could occur as a result of the disruption of some clusters under the action of evaporation provided they are tidally truncated (Surdin, 1994).

\section{The tidal limitation of the cluster size}

Globular clusters are fully relaxed systems whose external radius $\left(r_{t}\right)$ is limited by the galactic tidal field (Binney and Tremaine 1987):

$$
r_{t}=R_{p}\left[\frac{M}{2 g(e) M_{G}\left(R_{p}\right)}\right]^{1 / 3}
$$

where $\mathrm{M}$ is the cluster mass, $M_{G}\left(R_{p}\right)$ is the mass of the Galaxy inside the perigalactic distance of the cluster $\left(R_{p}\right)$, and $g(e)$ is a weakly verying function of the cluster orbit eccentricity $(e)$. A singular isothermal sphere 
is an adequate model of the Galaxy in our case:

$$
M_{G}\left(R_{p}\right)=\frac{R_{p} V_{c}^{2}}{G},
$$

where $V_{c}$ is the circular velocity. In this case, $g(e) \simeq 2$ (Seitzer, 1985). We can express $D_{0.5}$ in terms of $r_{h P}$, the radius containing half the cluster mass in projection:

$$
D_{0.5}=2 r_{h P}
$$

which simply depends on $r_{h}$, the half-mass radius of the cluster (Spitzer, 1987):

$$
r_{h P} \cong 0.74 r_{h},
$$

whose value can be expressed in terms of the core radius $\left(r_{c}\right)$ and the tidal cut-off radius for the King model (Fall and Rees, 1977):

$$
r_{h} \cong 0.70 \sqrt{r_{c} r_{t}}
$$

These give us the following simple relation:

$$
D_{0.5} \cong r_{t} 10^{-C / 2}
$$

where $C \equiv \lg \left(r_{t} / r_{c}\right)$ is a concentration index. Finally we obtain:

$$
D_{0.5} \cong\left(\frac{G M}{2}\right)^{1 / 3}\left(\frac{R_{p}}{V_{c}}\right)^{2 / 3} 10^{-C / 2}
$$

Considering the simplest case of circular orbits $\left(R_{g}=R_{p}\right)$ and equal concentrations $(C=1.5)$ of all clusters, we see that the connection between $D_{0.5}$ and $R_{g}$ depends on the value of $M$, which can not be arbitrary on the $D_{0.5}-R_{g}$ plane.

\subsection{AN UPPER LIMIT ON THE CLUSTER DIAMETER}

An upper limit on the cluster mass is determined by the observational limit of $M_{\max }=2.5 \cdot 10^{6} M_{\odot}$, which is caused by the natural exhaustion of the globular cluster luminosity function for large values of $M$. Then the restriction on the cluster diameter follows from the tidal stability condition (8):

$$
D_{0.5} \leq D_{\max }=8.5\left[\left(\frac{R_{g}}{1 k p c}\right)\left(\frac{220 \mathrm{~km} / \mathrm{s}}{V_{c}}\right)\right]^{2 / 3} p c
$$

which for $V_{c}=220 \mathrm{~km} / \mathrm{s}$ fits well the observable upper boundary of the cluster distribution on the $D_{0.5}-R_{g}$ plot (Fig. 1). 


\subsection{A LOWER LIMIT ON THE CLUSTER DIAMETER}

According to the results of numerical simulations the evaporation time of an isolated cluster is $t_{e v} \simeq 100 t_{r h}$, where $t_{r h}$ is the half-mass relaxation time. The evaporation is more rapid if the size of cluster is tidally limited: $t_{e v} \simeq(20-30) t_{r h}$. Taking into account that the physical diameter of a cluster reaches the tidal one only at the perigalactic point of their orbit, we can adopt $t_{e v}=70 t_{r h}$ as a reasonable compromise. According to the definition of $t_{r h}$, we obtain

$$
t_{e v}=\frac{1}{m}\left(\frac{M r_{h}^{3}}{G}\right)^{1 / 2},
$$

where $m$ is the mean stellar mass $\left(m=0.3 M_{\odot}\right)$. The evaporation of a cluster is very slow at the beginning, but accelerated to the end of the process. Then we can assume as a probable lower boundary for the cluster mass distribution the value of the mass obtained from equation (10) for a cluster evaporated during time $t_{e v}$ :

$$
M_{\min }=\frac{G M_{\odot}^{2} t_{e v}^{2}}{10 r_{h}^{3}} .
$$

Substituting $M_{\min }$ from equation (11) to equation (8), we obtain

$$
D_{\text {min }}=0.73 \cdot 10^{-C / 4}\left[\frac{G M_{\odot} t_{e v} R_{p}}{V_{c}}\right]^{1 / 3} .
$$

It is conventional lower limit on $D_{0.5}$ caused by cluster evaporation in the Galactic tidal field. For the simplest case of circular orbits and equal values of $C$ we obtain:

$$
D_{\min }=2\left[\left(\frac{R_{g}}{1 k p c}\right)\left(\frac{t_{e v}}{1.5 \cdot 10^{9} y r}\right)\left(\frac{220 k m / s}{V_{c}}\right)\right]^{1 / 3} p c
$$

which, for $t_{e v}=1.5 \cdot 10^{9} \mathrm{yrs}$ and $V_{c}=220 \mathrm{~km} / \mathrm{s}$, is near to observable lower boundary of the cluster distribution (Fig. 1).

\section{Evolutional? Yes! Primordial? Maybe.}

Even with very primitive assumption about circular orbits and equal values of $C$, the effects of the tidal truncation and evaporation of the clusters make it possible to predict the region of their localization on the $D_{0.5}-R_{g}$ plot. Besides, the upper and lower limits on the cluster diameters are power-law functions of $R_{g}$ with exponents of $2 / 3$ and $1 / 3$. Then the prediction for the 


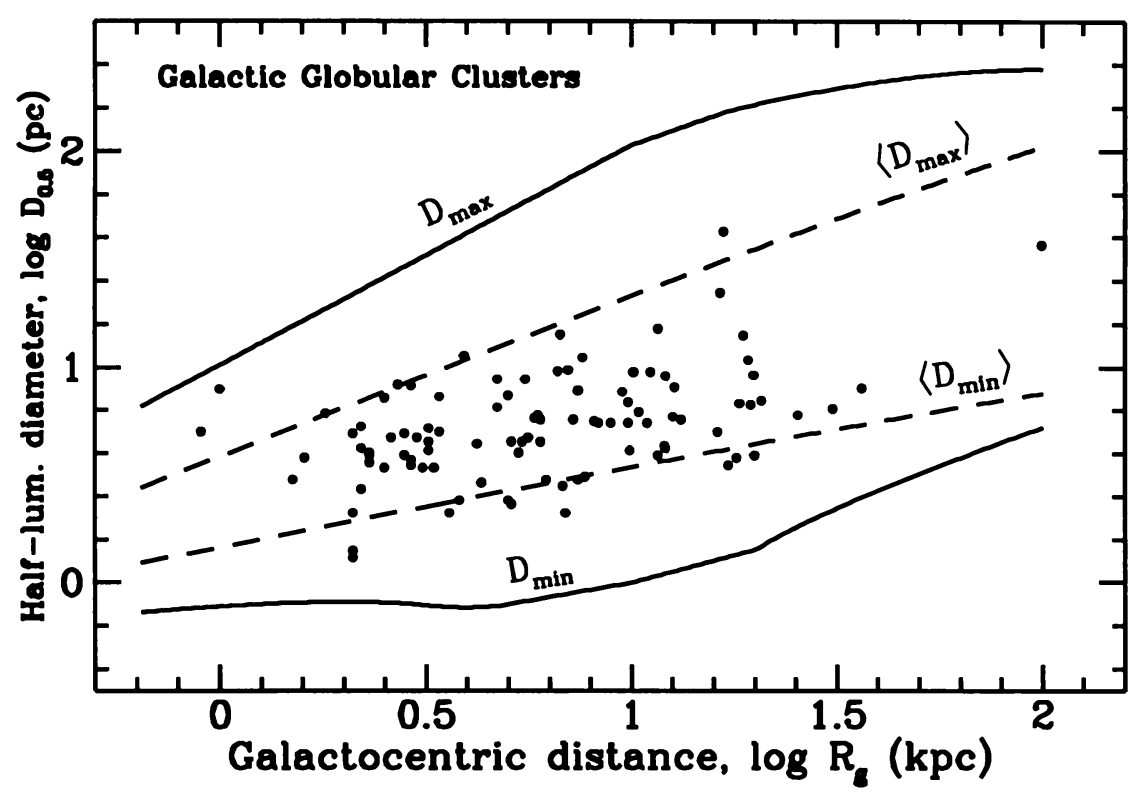

Figure 2. The same as in Fig.1, but for more realistic consideration.

relation between these two quantities is $D_{0.5} \propto R_{g}^{1 / 2}$. It looks absolutely like the observable correlation by van den Bergh et al. (1991).

We considered also these dynamical effects in more detail, taking into account the ellipticity of the cluster orbits and the distribution of their concentration parameters. We made the investigation like this and showed that the agreement of theoretical and observable boundaries becomes more precise, and the form of the correlation between $D_{0.5}$ and $R_{g}$ survives (Fig. 2). All clusters are located inside the absolute theoretical boundaries $\left(D_{\max }\right.$ and $\left.D_{\min }\right)$, and most of them are inside likelihood boundaries $\left(<D_{\max }>\right.$ and $\left.<D_{\min }>\right)$.

The similar limitation of globular clusters distribution one can obtain in plot "half-mass density - kinetic temperature" which is used in some scenarios of the clusters formation (Murray and Lin, 1992). In fact, dynamical evolution of any initial cluster population can explain this relationship.

Under development of this consideration, we found an absence of evolutional upper boundary fits observable distribution of clusters on plot "half- 
mass mean density - galactocentric distance", especially far from the Galactic center. This plot is most interesting for comparison of cluster formation theory with observations.

\section{Reconstruction of initial mass function}

In the Milky Way, as well as in M31, the number of clusters per unit mass for massive, well survived part of their population fits a simple powerlaw distribution: $N \propto M^{-2}$ (Surdin 1979; Racine 1980). Considering this spectrum as primordial one we can obtain initial total mass of the galactic globular cluster system: $M_{\text {tot }}(\mathrm{GC}) \sim 10^{8} M_{\odot}$ for the mass range of $6.5 \geq$ $\log \left(M / M_{\odot}\right) \geq 2.5$. It is order of $1 \%$ only from the stellar component of the galactic halo.

We can take into account a low efficiency of gravitationally bound systems formation. For the recent value of open cluster formation efficiency $(\mathrm{CFE} \approx 10 \%)$ extrapolated into the halo formation epoch, we can expect the total stellar mass connected with survived globular cluster population of $\sim 10^{9} M_{\odot}$. It is also less than the mass of stellar component of the galactic halo $\left(\sim 10^{10} M_{\odot}\right)$. Therefore we assume an existence of another population of low massive star clusters which was completely destroyed to the present. We use scenario by Sabano and Tosa (1983) for formation of protoclusters in slowly contracted turbulent Protogalaxy, and our scenario of evolution of gaseous protoclusters into stellar system (Surdin 1989). From this complex scenario of formation and early evolution of globular clusters we obtain good prediction for the total mass of the galactic halo (Surdin 1995): total mass of the halo stars $M_{H}=8 \cdot 10^{9} M_{\odot}$.

I wish to acknowledge the support of the LOC for my partisipance of the Symposium.

\section{References}

Binney, J. and Tremaine, S. (1987) Galactic Dynamics, Princeton Univ.Press.

Fall, S.M. and Rees, M.J. (1985) $A p$. J. 298, 18.

Murray, S.D. and Lin, D.N.C. (1992) Ap.J. 400, 265.

Racine, R. (1980) in Star Clusters, ed. J. E. Hesser, Reidel, Dordrecht, p. 369.

Sabano, Y. and Tosa, M. (1983) Theor. aspects on structure, activity and evolution of galaxies. University of Tokyo, p. 15.

Seitzer, P. (1985) in Dynamics of Star Clusters. IAU Symposium 113,eds. J. Goodman and P. Hut, Reidel, Dordrecht, p. 343.

Spitzer, L. (1987) Dynamical Evolution of Globular Clusters, Princeton University Press, New Jersey.

Surdin, V.G. (1979) Sov. Astron. 23, 648.

Surdin, V.G. (1989) Astron. Nachr. 310, 381.

Surdin, V.G. (1994) Astronomy Letters 20, 15.

Surdin, V.G. (1995) Astronomy Letters 21, N 4.

van den Bergh, S., Morbey, C. and Pazder J. (1991) Ap. J. 375, 594. 